\title{
Experimental Observation of Coherence and Stochastic Resonances in an Electronic Chua Circuit
}

\author{
Oscar Calvo*, Iacyel Gomes*, Claudio R. Mirasso* and Raúl Toral ${ }^{*}$, \\ * Departament de Física, Universitat de les Illes Balears, 07071-Palma de Mallorca, Spain \\ ${ }^{\dagger}$ Instituto Mediterráneo de Estudios Avanzados , CSIC-UIB, 07071-Palma de Mallorca, Spain
}

\begin{abstract}
Stochastic and coherence resonances appear in nonlinear systems subjected to an external source of noise and are characterized by a maximum response at the optimal value of the noise intensity. This paper shows experimentally that it is possible to observe them in a chaotic system. To this end we have analysed an electronic Chua circuit running in the chaotic regime and added noise to its dynamics. In the case of coherence resonance, we observe an optimal periodicity for the jumps between chaotic attractors, whereas in the case of stochastic resonance we observe a maximum in the signal-to-noise ratio at the frequency of an external sinusoidal perturbation.
\end{abstract}

\section{INTRODUCTION}

Contrary to the intuitive role of noise as a disordering agent, it has been shown that the response of a nonlinear dynamical system can improve in some occasions by the addition of noise. The paradigmatic and pioneering example is that of stochastic resonance in bistable systems $[1,2]$ which had been originally proposed as a mechanism for the observed periodicity of the Earth's ice ages. In this case, a dynamical system with two stable fixed points is subjected to the action of a periodic forcing and noise. The periodic forcing is sufficiently weak that, by itself, can not overcome the separation barrier between the stable fixed points and the system oscillates around one of them, chosen by the initial conditions. The presence of noise, however, allows the system to make random excursions and visit both fixed points. The jumps between the fixed points are easier to make when the effect of the external forcing is to lower enough the separation barrier. If the average time for the stochastic walk to go from one fixed point to the other coincides with the periodicity of the external signal, a maximum coincidence of the jumps with the external period is observed. The coincidence is optimal for the right value of the noise intensity. This is, basically, the mechanism for stochastic resonance. The original example has been extended to many other cases of physical as well as biological interest and there exists now a well established number of results concerning theoretical, numerical and experimental studies of stochastic resonance under a large variety of conditions [3, 4, 5].

A related but different phenomenon is that of coherence resonance by which a quasiperiodic signal is generated under the effect of the fluctuations in a system not subjected to a periodic forcing $[6,7,8]$. Again, the periodicity is optimal (resonance) for a given value of the noise intensity. The simplest example is an excitable system. This is a 
system with a fixed point which is stable for low amplitude perturbations. However, perturbations larger than a threshold value result in the system returning to the fixed point after a large excursion, a pulse, in phase space. In the case of coherence resonance, the perturbations are induced by noise and, if the noise has the appropriate intensity, the system is excited at the end of every pulse in such a way that a quasi-periodic train of pulses is generated. Coherence resonance has also been observed in bistable and oscillatory systems[9,10] or close to the onset of a bifurcation[11]. Experimental evidence has been shown for excitable electronic circuits[12, 13] and a laser system[14].

In this paper we show experimental evidence for both resonances in electronic Chua circuits operating in the chaotic regime. The possibility of having stochastic resonance in a chaotic circuit with two attractors under the presence of noise had been shown theoretically and numerically by Anishchenko and coworkers $[15,5]$. This is different from the so-called deterministic stochastic resonance[16] which appears in deterministic chaotic systems close to the onset of intermittency and that has been shown experimentally in ferromagnetic[17] and laser[18, 19] systems.

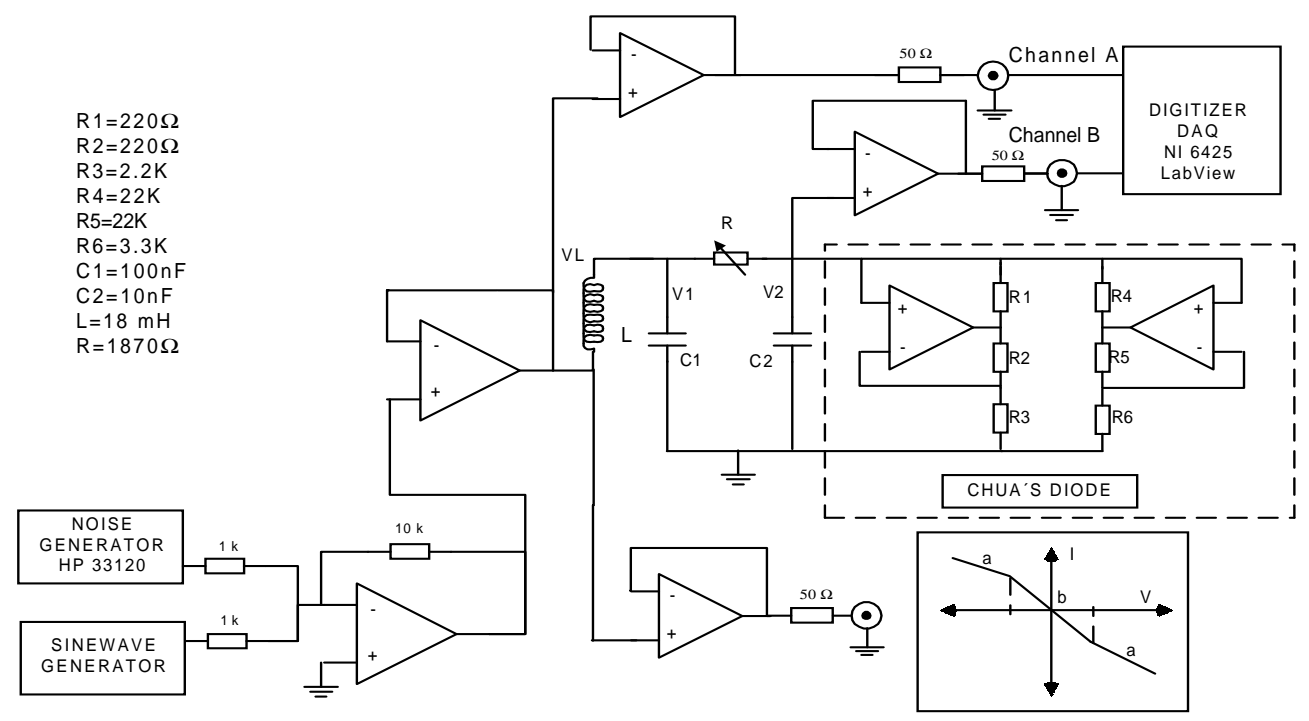

FIGURE 1 Experimental setup.

Our Chua circuit is shown in figure 1. A digital acquisition board from National Instruments NI-DAQ plugged into a laptop computer was used to digitize the signal at a sampling rate of $10 \mathrm{KHz}$. A Labview program controlled the board in a continuous acquisition mode and an HP 33120 function generator was used to provide the noise signals from 0 to $5 \mathrm{~V}$ [rms]. A Chua circuit can be described in terms of three non-linear first order differential equations[20]. These equations predict that the system has, for some range of parameters, three unstable fixed points. In this case, the output voltages $V_{1}$ and $V_{2}$ oscillate chaotically in time around different dynamical attractors. In our experiments, we choose the main parameters $C_{1}=10 \mathrm{nF}, C_{2}=100 \mathrm{nF}, R=1673 \Omega$, $a=-8 / 7, b=-5 / 7$, where $a$ and $b$ characterize the slope of the non-linear Chua resistance (see inset fig. 1). For this set of parameters, there is a single scroll attractor (as shown in the fig. 2(a), and its mirror image (not shown in the figure). In the absence 
of any external perturbation, $V_{1}$ and $V_{2}$ chaotically oscillate around one of the unstable fixed points with a mean oscillation frequency of $\sim 2.3 \mathrm{KHz}$ and without any possibility to jump to its mirror image attractor. Depending on the initial conditions, the system moves within this attractor or on its mirror image. The time evolution of $V_{2}$ is shown in the figure 2(b). We can see that $V_{2}$ (and similarly for $V_{1}$ ) oscillates chaotically with a rather large fluctuations in the minima and maxima values. This fact will be important when studying the stochastic and coherence resonance phenomena, as will be discussed later.
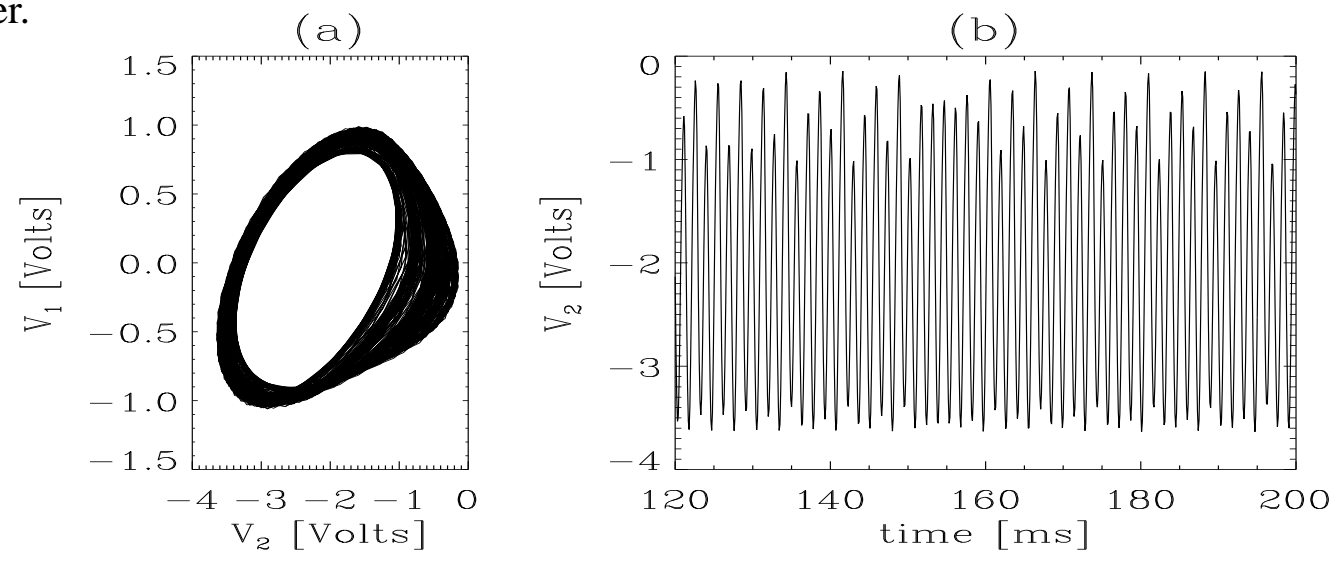

FIGURE 2 (a) Phase space representation of the attractor; (b) Time series of $V_{2}$

To study the stochastic resonance effect we start by adding a small periodic signal (a sinusoidal one) to the circuit in the branch of the impedance $L$ as shown in figure 1 . The amplitude of the signal is sufficiently small $(\sim 20 \mathrm{mV})$ that the system is not able to jump to its mirror image attractor. The frequency of the signal is $\sim 2.1 \mathrm{KHz}$, close to the mean frequency of the Chua attractor. A white noise source is also added to the system through the same branch of the periodic signal source. We have checked that in the absence of the periodic signal, the system remains in the same attractor, and does not jump to its mirror image, for small noise levels. Once the noise is increased the system starts to jump from one attractor to the other. This situation is depicted in Figure 3(a), where we plot the time evolution of $V_{2}$ for three different noise levels, together with the sinusoidal signal. In a typical stochastic resonance behavior, e.g. when a system jumps between two potential wells with stable minima, its is expected that an optimum noise level exists for which the system jumps at every external signal period. In our case, however, we do not move between two stable minima but between two chaotic attractors, each with its own internal dynamics. When looking in more detail at the jumping process between the attractors, we observe that after a jump the system starts to move close to the center of the attractor and not in the outer orbits. In this case it is unlikely that the system would jump to the other attractor until it reaches an outer orbit. Therefore, for the sinusoidal frequency we have chosen, we do not expect a jump from one attractor to the other at every sinusoidal period. This fact is also reflected in figure 3(a). The center panel corresponds to the time trace of $V_{2}$ with the optimum noise level. Although the resonance between the noise and the external periodic signal is not clear, it becomes evident when plotting the Fourier transform of the signal, $|S(v)|$ (power spectrum). In figure 3(b) it can be observed a maximum of the Signal-to-Noise Ratio (SNR) at an intermediate noise level, in this case 
a value of $\sim 1.5 \mathrm{~V}$ [rms]. This fact is confirmed when plotting the SNR vs. the noise level (see figure 4). A clear maximum appears indicating an optimum response of the system to this intermediate noise intensity.
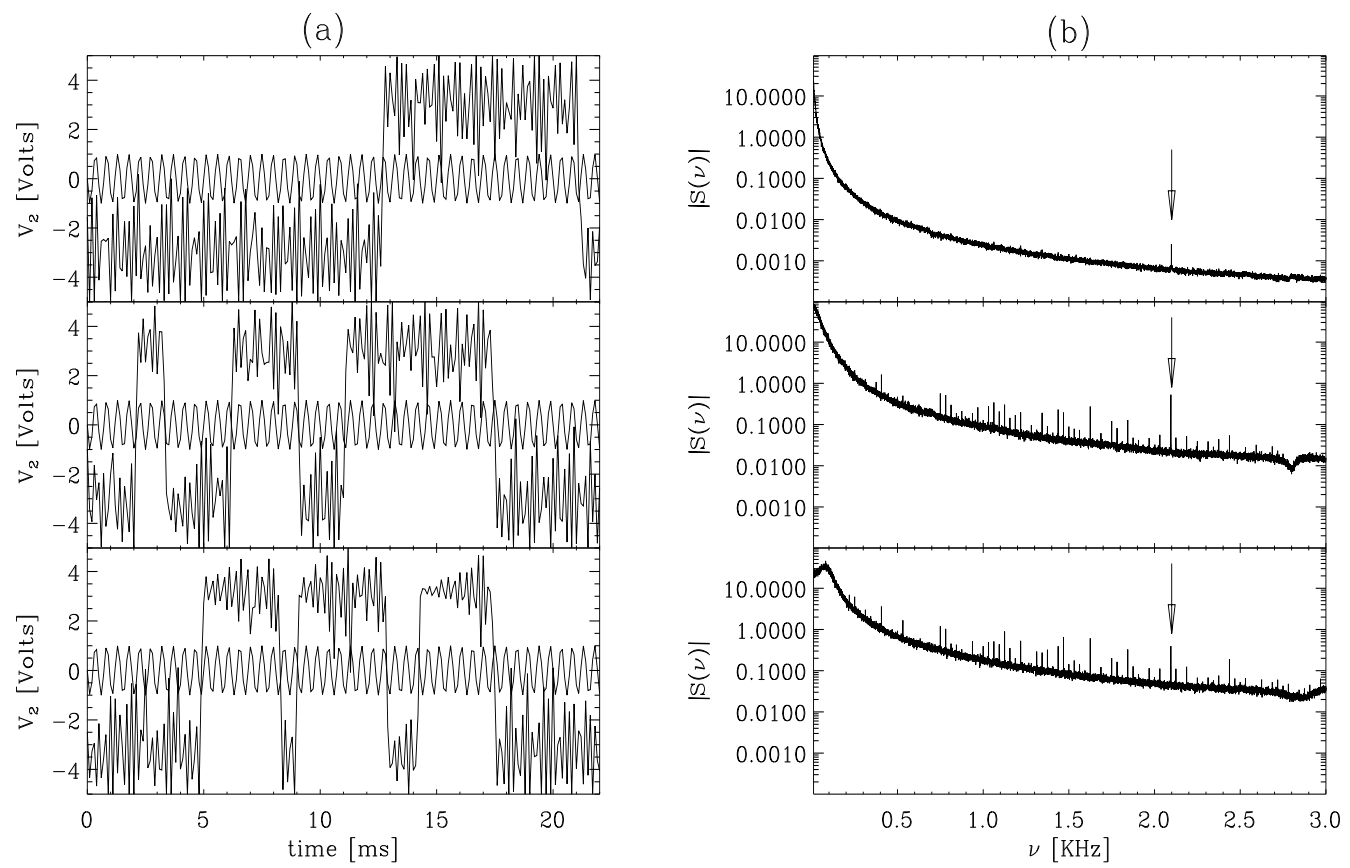

FIGURE 3 (a) Time evolution of $V_{2}$ for three different noise levels. From top to bottom: $1 \mathrm{~V}$ [rms], $1.5 \mathrm{~V}$ [rms] and $2.25 \mathrm{~V}$ [rms]. The sinusoidal signal is added in the three panels with an arbitrary amplitude; (b) Power spectrum for the same noise intensity as in panel (a). The arrow indicates the frequency of the sinusoidal signal.

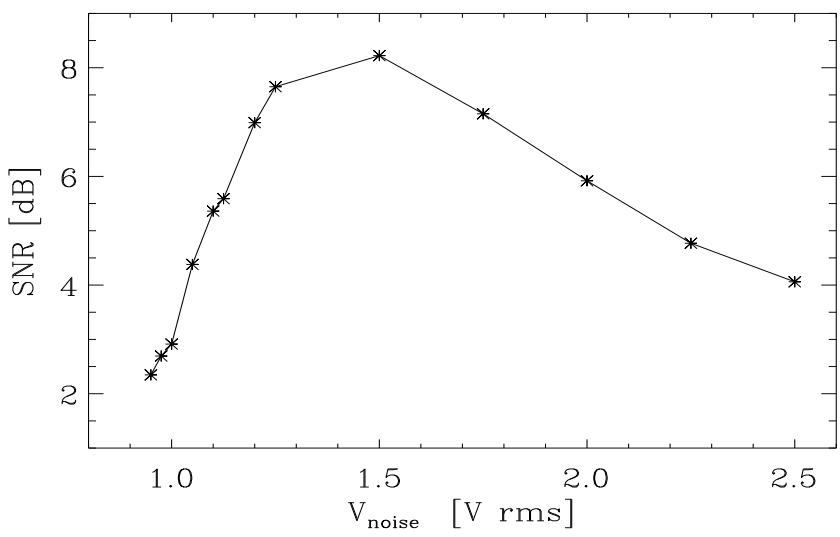

FIGURE 4 Signal-to-noise ratio as a function of the noise level.

The phenomenon of coherence resonance is characterized by an optimum response of a system for an intermediate noise level without any other forcing. In this sense it is not very different to the stochastic resonance except for the absence of the sinusoidal source, 
and for this reason it is sometimes called stochastic resonance without external forcing. We start our experiment in the same situation as we started the previous one. Again for small noise levels the system is not able to jump to its mirror image attractor. As we do not have a periodic external forcing we do not expect any resonance in the SNR. This means that we have to look for other indicators. To quantify the situation we calculate the variance $\sigma$ of the residence time in the attractors normalized to the mean value $\langle t\rangle$ and the normalized autocorrelation function of the time series[8]. Three typical time traces are shown in the figure 5(a) for the voltage $V_{2}$ at low, intermediate and large noise levels. It can be seen that for the intermediate noise level (the optimum one), the regularity of the jumping process becomes evident. This is the first indication that noise is inducing a more regular behavior in this chaotic system. The autocorrelation functions depicted in figure 5(b) present the deepest minimum value at the same noise level, indicating maximum anticorrelation between the two states. These results reveal the existence of a constructive effect of the noise that is capable to yield a maximum regularity in the process of transition from one chaotic attractor to the other.
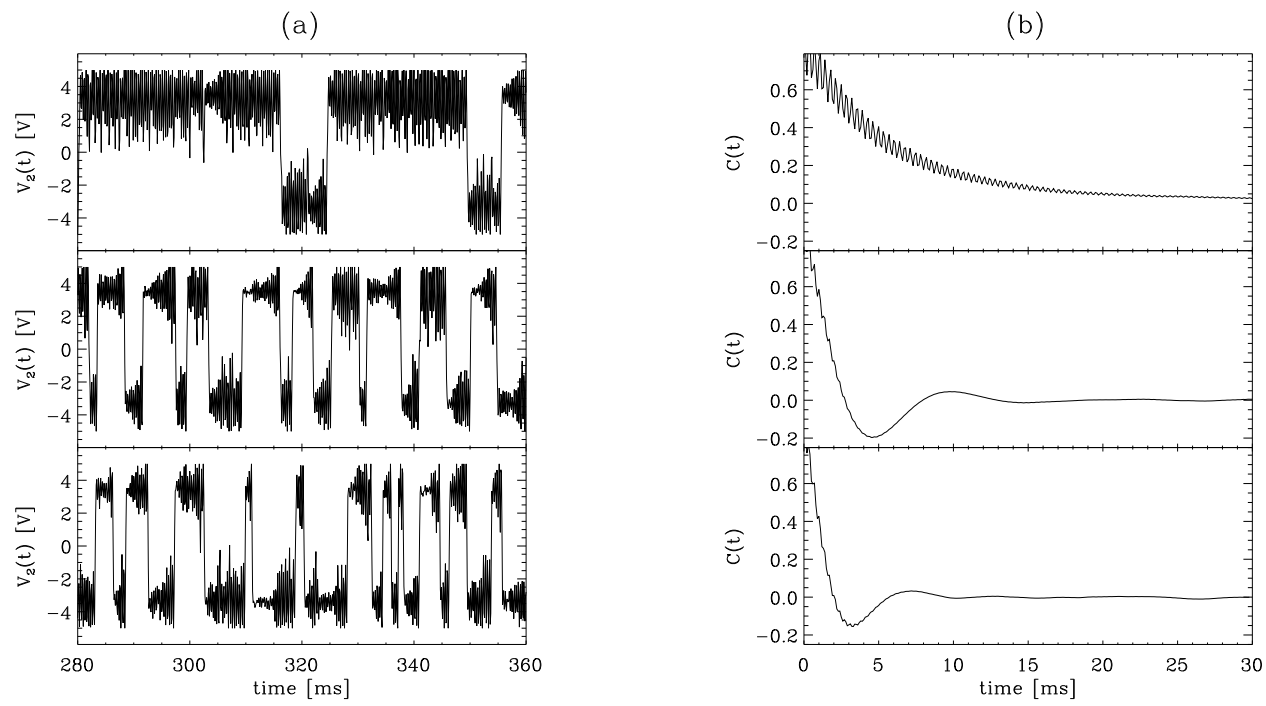

FIGURE 5 (a) Voltage time series for three different noise levels (maximum values): 0.5 $\mathrm{V}[\mathrm{rms}]$, 1.5 V[rms], optimum noise level, and 2.5 V[rms]; (b) Autocorrelation function for the same noise levels.

Finally, in figure 6 we plot both the normalized variance of the residence time and the minima of the autocorrelation functions vs. the noise levels. Both quantities have a minima close to the noise intensity $\sim 1.5 \mathrm{~V}[\mathrm{rms}]$.

In conclusion, we have experimentally shown that the main features of both stochastic and coherence resonance are present in some chaotic systems. We have done the demonstration using a chaotic Chua circuit. In the case of the stochastic resonance we observed a clear maximum for an intermediate noise level of the signal-to-noise ratio at the external frequency. For the coherence resonance, a quasi-periodic response can be induced by just applying an appropriate amount of noise to a circuit which, otherwise, exhibits a non-regular behavior. The quality of the periodicity was quantified by the variance of the residence time and the minimum of the time correlation function. 


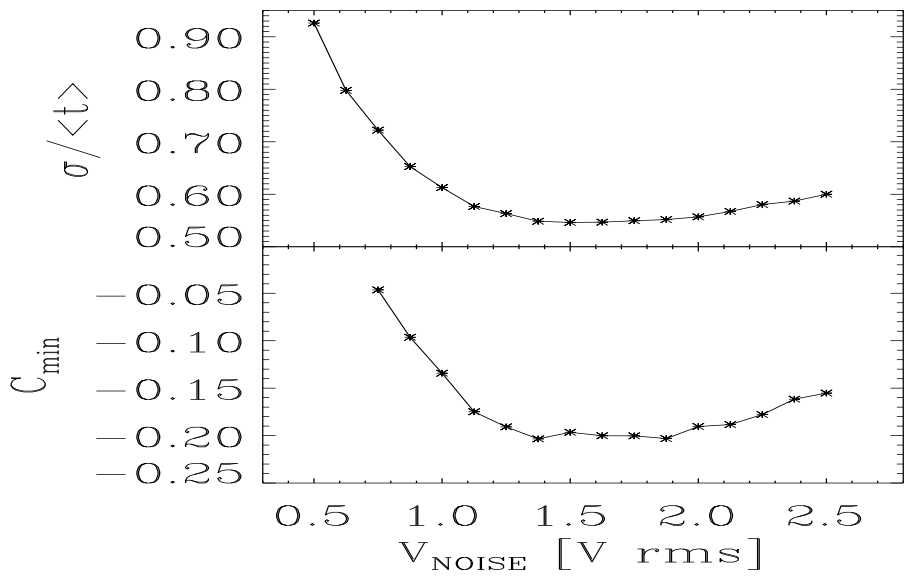

FIGURE 6 (a) Variance of the residence time normalized by the mean time $\sigma /\langle T\rangle$ and (b) minimum of the correlation function $C_{\min }$ as a function of the noise level $V_{N O I S E}$.

We thank financial support from DGESIC (Spain) projects numbers PB97-0141-C0201 and BFM2000-1108.

\section{REFERENCES}

1. R. Benzi, A. Sutera and A. Vulpiani, J. Phys. A14, 453 (1981).

2. C. Nicolis and G. Nicolis, Tellus 33, 225 (1981).

3. Proceedings of the NATO Advanced Research Workshop: Stochastic Resonance in Physics and Biology. F. Moss, A. Bulsara and M.F. Shlesinger, eds. J. Stat. Phys. 70 (1993).

4. L. Gammaitoni, P. Hänggi, P. Jung and F. Marchesoni, Rev. Mod. Phys. 70, 223 (1998).

5. V.S. Anishchenko, A.B. Neiman, F. Moss and L. Schimansky-Geier, Physics-Uspekhi 42, 7 (1999).

6. H. Gang, T. Ditzinger, C.Z. Ning and H. Haken, Phys. Rev. Lett. 71, 807 (1993).

7. W. Rappel and S. Strogatz, Phys. Rev. E50, 3249 (1994).

8. A.S. Pikovsky and J. Kurths, Phys. Rev. Lett. 78, 775 (1997).

9. B. Lindner and L. Schimanwky-Geier, Phys. Rev. E 61, 6103 (2000).

10. J.R. Pradines, G.V. Osipov and J.J. Collins, Phys. Rev. E 60, 6407 (1999).

11. A. Neiman, P. Saparin and L. Stone, Phys. Rev. E 56, 270 (1997).

12. D.E. Postnov, S.K. Han, T.G. Yim and O.V. Sosnovtseva, Phys. Rev. E59, R3791 (1999).

13. S.K. Han, T.G. Yim, D.E. Postnov and O.V. Sosnovtseva, Phys. Rev. Lett. 83, 1771 (1999).

14. G. Giacomelli, M. Giudici, S. Balle and J.R. Tredicce, Phys. Rev. Lett. 84, 3298 (2000).

15. V.S. Anishchenko, M.A. Safanova and L.O. Chua, Int.J. Bif. Chaos 2, 397 (1992).

16. V.S. Anishchenko, A.B. Neimann and M.A. Safanova, J. Stat.Phys. 70, 183 (1993).

17. E. Reibold, W. Just, J. Becker and H. Benner, Phys. Rev. Lett. 78, 3101 (1997).

18. A. N. Pisarchik and R. Corbalán, Phys. Rev. E58, R2697 (1998).

19. V.N. Chizhevsky, R. Vilaseca and R. Corbalán, Phys. Rev. E61, 6500 (2000).

20. Chua's Circuit: A Paradigm for Chaos, R.N. Madan, ed. World Scientific Publishing (1993). 